\title{
Induction of distinct gene expression patterns in lymphoid and epithelial cells by the BARF-I gene of Epstein-Barr virus
} A Zur Hausen*1, T Wiech ${ }^{1}$, E Nikolopoulos ${ }^{1}$, S Lassmann ${ }^{1}$, T Heidt ${ }^{1}$, M Sarbia ${ }^{2}$, A Walch ${ }^{2}$, M Werner ${ }^{1}$ and T Ooka ${ }^{3}$

Address: ${ }^{1}$ Institut für Pathologie, Universitätsklinikum Freiburg, Germany, ${ }^{2}$ Technische Universität München, Institut für Pathologie, Germany and ${ }^{3}$ Université Claude Bernard Lyon, Laboratoire de Virologie Moléculaire, Lyon, France

* Corresponding author

from 35te Tagung der Pathologen am Oberrhein/35th Meeting of Pathologists of the Upper Rhine Region (PATOR)

The Institute of Pathology, University Hospital Freiburg, Germany. I July 2006

Published: I4 March 2007

Diagnostic Pathology 2007, 2(SuppI I):S9 doi:I0.1 I86/I746-I596-2-SI-S9

(C) 2007 Zur Hausen et al; licensee BioMed Central Ltd.

\section{Background}

The expression of the BARF1 gene of Epstein-Barr virus (EBV) in latent EBV infection is restricted to epithelial malignancies, e.g. gastric carcinomas (GC) or nasopharyngeal carcinomas (NPC). In addition, BARF1 is considered to be a lytic gene because it is expressed upon induction of the lytic cycle in Burkitt's lymphoma cell lines.

\section{Aims}

To analyze the gene expression patterns of a BARF1-transfected epithelial and lymphoid cell line in order to identify cellular genes regulated by BARF1.

\section{Methods}

Gene expression of a BARF1-transfected lymphoblastoid (Louckes+) and a BARF1-transfected epithelial (HaCaT+) cell line were compared by cDNA microarray analysis using Affymetrix U133A chips. Of each group, 6 genes were confirmed by Realtime PCR. In addition, immunohistochemistry (IHC) and fluorescence in situ hybridization (FISH) was performed on a tissue microarray (TMA) of 181 gastric carcinomas for selected genes, including 11 EBV-associated gastric carcinomas $(6.1 \%)$ as tested by EBER-RNA in situ hybridization.

\section{Results}

The Louckes+ cells revealed 730 genes downregulated and 545 genes were upregulated, whereas the number of upregulated genes in epithelial HaCaT+ was 10-fold lower and the number of downregulated genes comparable with Louckes+. Among others, cyclin D1 expression was signif- icantly upregulated in HaCaT+ by BARF1, but not in Louckes+. Analyzing cyclin D1 expression on a TMA of 181 gastric carcinomas revealed a significant overexpression of cyclin D1 in EBV-associated gastric carcinomas ( $\mathrm{p}$ $<0.012$ ) but not in EBV-negative gastric carcinomas as tested by IHC. Cyclin D1 FISH showed that cyclin D1 overexpression was not due to gene amplification.

\section{Conclusion}

Expression of BARF1 induces the expression of distinct sets of cellular genes in epithelial and lymphoblastoid cells underlining the restricted BARF1 expression patterns in latent epithelial EBV infection and lytic EBV replication in lymphoblastoid cell lines. In addition, the overexpression of cyclin D1 in EBV-associated gastric carcinomas is induced by BARF1 and not due to cyclin D1 gene amplification. Next to cyclin D1, other genes have been identified in this study by cDNA microarray analysis which might help to understand the role of EBV in gastric carcinogenesis. 